\title{
Changing the Culture of Clinical Education in Massage Therapy
}

\author{
Amanda Baskwill, BEd, RMT \\ Humber College Massage Therapy Program, Humber College, Toronto, Ontario, Canada
}

Much within the profession of massage therapy is done according to tradition. From an epistemological viewpoint, tradition is a way of knowing or, by extension, being, that is based upon both tenacity and authority and not always in best practices. As the profession of massage therapy moves in the direction of evidence-based medicine, or evidenceinformed practice, the opportunity to re-evaluate massage therapy education presents itself.

KEYWORDS: clinical education, massage therapy, outcomes-based

\section{INTRODUCTION}

Much within the profession of massage therapy is done according to tradition. From an epistemological viewpoint, tradition is a way of knowing or, by extension, being, that is based upon both tenacity and authority and not always in best practices ${ }^{(1)}$. As the profession of massage therapy moves in the direction of evidence-based medicine, or evidence-informed practice $^{(2)}$, the opportunity to re-evaluate massage therapy education presents itself.

The traditional approach to massage therapy education has been for massage therapy students to learn specific 'recipes' by which to treat patients. In practice, no patient is exactly the same as the one for whom the recipe was created. Jones and Rivett ${ }^{(3)}$ recognize that education for manual therapists, including massage therapists (MTs), must support the development of clinical reasoning skills so MTs can create and adapt care to environments, scenarios, and patient presentations. The main purpose of health care practitioners' education, therefore, can be seen as turning unskilled novices into confident, skilled professionals ${ }^{(4)}$.

Higgs and Jones ${ }^{(5)}$ define clinical reasoning as "a process incorporating the elements of cognition, knowledge, and metacognition". Essentially, it is clinical reasoning that enables a health care professional to be autonomous. These authors suggest that clinical reasoning also creates responsibility, accountability, and confidence in decision-making in difficult or uncertain situations in those who develop these skills. It was with these goals in mind that the faculty of massage therapy at Humber College, in Toronto, Ontario, set out to shift the culture of clinical education in massage therapy.

\section{METHODS}

\section{An Innovative Approach to Clinical Education}

In the massage therapy program at Humber College it is believed that clinical courses have the possibility to provide experiences for Student Massage Therapists (SMTs) that develop clinical reasoning and alter the professional self-image of SMTs while developing their professional competence. As a result of setting clinical reasoning at the center of the clinical encounter, the MT faculty has created an innovative approach to clinical education.

Anecdotal reports from previous clinical placements indicate that both instructors and students believe patients' desire simply to have a massage, a spa-like environment, and that students tend to confine their learning to only the time when they are in the clinic area. These factors affect the usefulness of clinical placements. It is believed by the faculty that the value of clinical education is too important to the overall development of Student Massage Therapists to allow these elements to undermine the productivity of these opportunities. As a result, when developing the program at Humber College in 2009, an effort was made to incorporate strategies to positively affect these elements that traditionally occur in MT clinical placements. Three key strategies that have been incorporated specifically into the program are: taking an outcomes-based approach to treatment, creating a health care environment and attitude, and incorporating an academic/theoretical approach to clinical placement curriculum.

\section{Outcomes-based Approach to Treatment}

The approach taken to practice in Outcomes-based Massage is one in which SMTs are required to work with a patient through four phases of treatment including evaluative, treatment planning, treatment, 
and discharge ${ }^{(6)}$. This is a particular approach to clinical decision-making that encourages SMTs to consider what they do and why. It also requires practitioners to link their assessment findings to the treatment goals and the techniques or modalities used. This comprehensive approach to care ensures that treatment is given with a therapeutic purpose. An outcomes-based approach to massage therapy encourages clinical reasoning, and gives SMTs tools rather than recipes.

Each new patient begins his or her treatment in the Student Massage Therapy Clinic with an initial visit, primarily consisting of an initial assessment. Regardless of presenting complaint, each is assessed for vital signs and posture. Any subsequent assessment is focused on the patient's goals for treatment and clinical presentation. Following the evaluative phase, SMTs create an individualized treatment plan consisting of short and long-term goals, duration and frequency of treatment, the length of the treatment plan, techniques, modalities, self-care and referrals.

Once a treatment plan is established, SMTs provide treatment according to the plan. They are expected to conduct a relevant assessment before and after each treatment to monitor patients' short and long-term progress. At the conclusion of the treatment plan, a formal re-evaluation of the patient is done. Where progress is seen, SMTs either discharge the patient to self-care or create a new treatment plan for the next phase of treatment. Where no progress has been seen, the treatment plan is evaluated and either modified or the patient is referred to another health care professional for care.

It is anticipated that this approach to massage therapy practice will encourage student massage therapists to think critically as they assess a patient and determine a treatment plan. By linking techniques and modalities to outcomes, SMTs have the opportunity to test out their clinical reasoning skills as they evaluate whether or not their plan is having its intended effect. All of this occurs in an environment that supports student learning through the opportunity of clinical placement.

\section{Health Care Environment and Attitude}

Clinical education is important in the development of professional competence ${ }^{(7)}$. However, it is only as useful as the opportunities created for learning to occur. These learning opportunities are supported by the environment of the treatment area and the attitudes of the instructors and students which, in turn, influence the attitudes and expectations of patients.

In many massage therapy student clinics, treatment provided by SMTs occurs behind closed curtains where clinical instructors must ask permission to enter the treatment area and, once in, discover whether or not a teachable moment exists. Similarly, in many massage therapy student clinics, the lights are turned off and soft music is playing, creating a spa-like atmosphere. While massage therapists may choose to set up their practices in this way, it is not surprising that patients, in student massage therapy clinics that create this type of environment, want to avoid assessment and proceed directly to the technique portion of the appointment.

Since the program at Humber College seeks to support the evolution of massage therapy as it becomes more accepted as the health care profession it is, the faculty has worked to develop an atmosphere that supports student education and patient safety. The environment that has been created is clinical, patients have been educated as to the purpose of the clinic and their role within it, and a fee schedule has been created that focuses on the outcomes-base approach to massage therapy as described above, with the intent of creating an environment and attitude that supports student clinical education.

To create an environment that is clinical in nature and supports student learning, the student clinic keeps the lights on at all times and does not play music, and the fronts of the treatment areas are open, allowing for the clinic instructor to observe the SMT at all times. It is believed that by keeping the lights on, students are able to engage fully in each part of the appointment. For example, a SMT with a new patient can take the time he or she needs to conduct a full initial assessment without having to do part of it in the dark. Students who are applying trigger point techniques can clearly see any tissue response. The open treatment areas allow clinical instructors to provide feedback to students without interrupting what they are doing. It also ensures that, should an SMT be performing something unsafely, immediate intervention can occur.

An additional strategy to alter attitudes about massage therapy student clinics that has been employed is a patient information brochure. This brochure contains information about the development and purpose of the clinic, and describes to patients what to expect during their appointment. It also contains the clinic policies such as no tipping and reduced scent, and conflict of interest policies. This brochure is sent to every patient when they book an appointment in the clinic.

Also provided in the clinic is a fee schedule. Following the College of Massage Therapists of Ontario requirements, the schedule of fees for the student clinic is posted in the reception area and available for distribution to the patients ${ }^{(8)}$. Four types of appointments have been developed and each is described on the fee schedule. All new patients participate in an initial appointment. This appointment focuses on gathering health history information, conducting physical assessments, and establishing goals for treatment and a treatment plan. Once a treatment plan has been established, patients book in for a subsequent appointment. In a subsequent appointment, patients 
receive treatment and assessment according to their established treatment plan. Returning patients who have a new concern or injury book an appointment for a new treatment plan. In a new treatment plan appointment, patients are assessed for the new presentation and a new treatment plan is created. Finally, the fourth type of appointment is a postdischarge assessment. Once a patient is discharged, they are invited in for a subsequent assessment. This assessment determines how they have been doing subce the treatment plan was concluded. This appointment has no charge.

The strategies used in establishing a clinical environment and attitude toward massage therapy practice are not time-consuming and can be used by students, once they are in practice, to support their continued use of a clinical approach to treatment.

\section{An Academic Approach to Clinical Education}

Within clinical placements, interactions with patients are not useful for developing professional competence when the students' tendencies are to confine the teachable moment to the time actually spent in the treatment area. Often, it is not part of massage therapy clinical education to contemplate treatment outside of designated course time. It is the belief of the faculty in Humber College's program that when this is allowed within entry-to-practice education, it is the behavior that continues to be followed once the individual is in professional practice. As such, more conscientious values should be encouraged.

To achieve the goal of encouraging the values of clinical reasoning, critical thinking, and evidenceinformed practice, a more structured approach to clinical courses has been designed. Within this approach — which one might call an academic approach to clinical education - three distinct strategies have been embedded in the students' clinical experience: learning modules, case presentations, and clinical follow-ups.

Learning modules are predetermined learning strategies and activities that the students are required to complete as part of their evaluation for clinical placement. Depending on the level and focus of the clinical placement, the learning modules differ. For example, in the first clinical placement in the program that occurs in the third of six semesters, students focus on developing therapeutic relationships and boundaries, as well as their treatment planning skills. Some of the learning modules facilitate SMTs' exploration of these concepts within their clinical practice. Other learning modules focus on skills SMTs have learned in their theory courses that they are now expected to demonstrate in clinic. This allows the SMT to direct his or her own learning, especially when the clinical instructor may be busy helping another student.

Case presentations are a concept borrowed from clinical education in other health care professions ${ }^{(9)}$.
For this element, students are asked to verbally present their patients to the clinical instructor. This is a formal presentation in which students are expected to describe all relevant information about the patient to the instructor. Based on the information presented, the instructor then asks a series of questions challenging the student to think critically about what they have discovered or what they have intended in their treatment plan. Often this drives the student to conduct further assessment, or helps to identify an area of knowledge or skill the student is lacking. It is then through the third strategy that students fill that gap.

Clinical follow-ups are the final strategy specific to an academic approach. Clinical follow-ups stimulate the development of knowledge and skills that students are lacking that are specific to the treatment of a particular patient. It is through independent study or information-gathering that students find information and practice skills, and develop memory aids or similar strategies to ensure they are competent to treat the patient in the next appointment. Common topics include knowledge of medication, development of an assessment skill, practice of a technique or modality, evidence for their proposed treatment plan, and knowledge of a medical condition. Students receive credit for these clinical follow-ups in the evaluation structure for the course.

It is the intention of the faculty in the Humber College Massage Therapy program that the inclusion of these strategies facilitates the development of the knowledge, skills and dedication required for student massage therapists to take a critical, reasoned, and evidence-informed approach to practice.

\section{CONCLUSION}

Evolution naturally occurs in professions as external, or internal, influences put pressure for change on the current culture. Massage therapy has the opportunity to embrace some of the current external pressures, such as a move toward evidence-informed practice, and to place internal pressure on the current culture of massage therapy to stimulate change. It is the hope of the faculty in the Massage Therapy program at Humber College that, by creating change within the education of student massage therapists, the profession of massage therapy will be allowed to evolve. The next phase of evolution will hopefully be one in which massage therapists are active, competent, and valued members of a health care team-members who have skills in clinical reasoning and take an evidence-informed approach to care.

\section{COPYRIGHT}

Published under the CreativeCommons AttributionNonCommercial-NoDerivs 3.0 License. 


\section{REFERENCES}

1. Portney LG, Watkins MP. Foundations of clinical research: applications to practice. Toronto: Prentice Hall; 1993.

2. Sackett DL, Straus SE, Richardson WS, et al. Evidence-based medicine: how to practice and teach EBM, 2nd ed. New York: Churchill Livingstone; 2000.

3. Jones MA, Rivett DA, editors. Clinical reasoning for manual therapists. London: Butterworth-Heinemann; 2004.

4. Boshuizen HPA, Schmidt HG. The development of clinical reasoning expertise. In: Higgs J, editor. Clinical reasoning in the health professions, 3rd ed. London: Elsevier; 2008. p. 113-122.

5. Higgs J, Jones MA. Clinical decision making and multiple problem spaces. In: Higgs J, editor. Clinical reasoning in the health professions, 3rd ed. London: Elsevier; 2008. p. 3-17.

6. Andrade CK, Clifford P. Outcomes-based massage: from evidence to practice, 2nd ed. New York: Lippincott, Eilliams \& Wilkins; 2008.
7. Wong J, Wong S. Towards effective clinical teaching in nursing J Adv Nurs. 1987;12(4):505-13.

8. College of Massage Therapists of Ontario. Communication and public health standard 2: inform the client of the fees and obtain his/her agreement to a fee schedule [Internet]. Toronto: In house; 2006 [cited 2011 May 11]. Available from: http:// www.cmto.com/pdfs/CPH\%202.pdf

9. Onishi $\mathrm{H}$. The role of case presentation for teaching and learning activities. Kaohsiung J Med Sci. 2008;24(7):356-60.

Corresponding author: Amanda Baskwill, BEd, RMT, Humber College Massage Therapy Program, Humber College, 205 Humber College Blvd, Toronto, Ontario, Canada, M9W 5L7.

E-mail: amanda.baskwill@humber.ca 